\title{
Berechnungsmodell für die Elektrodialyse von Salzlösungen
}

\author{
Jens-Uwe Gerking, Josef Schmadl
}

Elektromembranverfahren spielen eine wichtige Rolle in natuirlichen Vorgängen, wie z. B. bei der Ernährung von Gehirnzellen, und sind von der Solarenergienutzung (elektrolytische Wasserstofferzeugung) und der elektrischen Energieerzeugung (PEM-Brennstoffzelle) uiber Abwasserreinigung, Wasserentsalzung und Wertstoffrückgewinnung (Elektrodialyse mit bipolaren Membranen oder mit Ionenaustausch) bis hin zum Einsatz in chemischen Syntheseverfahren auch technisch vielseitig einsetzbar. Sie sind aber noch wenig bekannt und längst nicht ausreichend erforscht. Mit dieser Arbeit soll ein Beitrag geleistet werden zur mathematischen Modellierung der Elektrodialyse mit Ionenaustauschermembranen.

\section{Prinzip der Elektrodialyse}

Eine Elektrodialyseapparatur mit Ionenaustauschermembranen besteht aus einer Folge von Kammern, die alternierend durch Anionen- und Kationenaustauschermembranen getrennt sind, wie in Abb. 1 dargestellt. Über zwei Elektroden an beiden Enden kann ein elektrisches Feld quer zur Durchströmungsrichtung angelegt werden. Unter seinem Einfluss wandern in den sog. Diluatkammern die Kationen durch die Kationenaustauschermembran (K) und die Anionen durch die Anionenaustauschermembran (A) in die jeweils benachbarten sog. Konzentratkammern. Ihre Weiterwanderung in Richtung Anode bzw. Kathode wird durch die nachfolgenden Ionenaustauschermembranen mit entgegengesetzter Ladung verhindert. Auf diese Weise wird ein Feedstrom in ein weitgehend entsalztes Diluat und ein Konzentrat aufgespalten. Die Grundeinheit besteht somit aus einer Diluat- und einer Konzentratkammer.

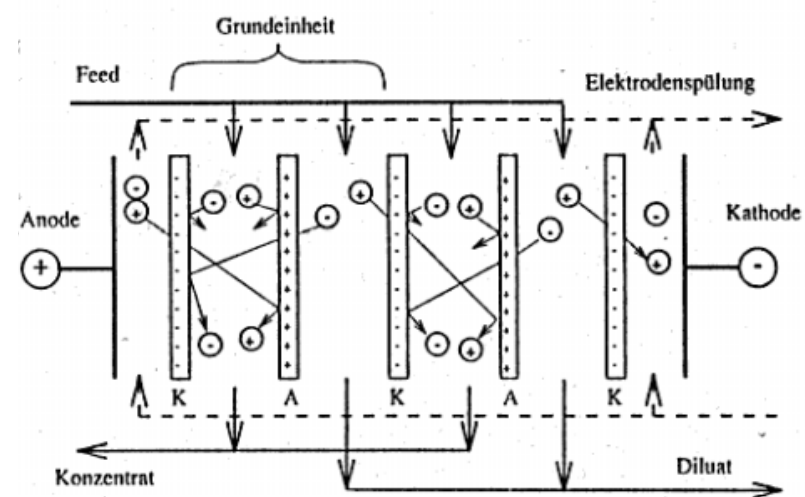

Abb. 1: Prinzipschema der Elektrodialyse mit alternierend angeordneten Ionenaustauschermembranen

In Abb.1 ist das kontinuierliche Verfahren dargestellt. Die Messungen in dieser Arbeit wurden jedoch in einer diskontinuierlich betriebenen Apparatur durchgefuihrt, bei der Vor- und Rücklauf jeweils für Diluat, Konzentrat und Elektrodenspülung über einen Vorratbehälter kurzgeschlossen sind und mit einer Pumpe in geschlossenem Kreislauf umgepumpt werden. Mit fortschreitender Elektrodialysezeit reichert sich dabei im Konzentratkreislauf das Salz an, im anderen, dem Diluatkreislauf, fällt die Konzentration.

\section{Theoretisches Modell}

Bei dem im folgenden dargestellten Berechnungsansatz wird angenommen, dass sich die Elektrodialyse von Salzen mit dem Beladen eines Kondensators vergleichen lässt.

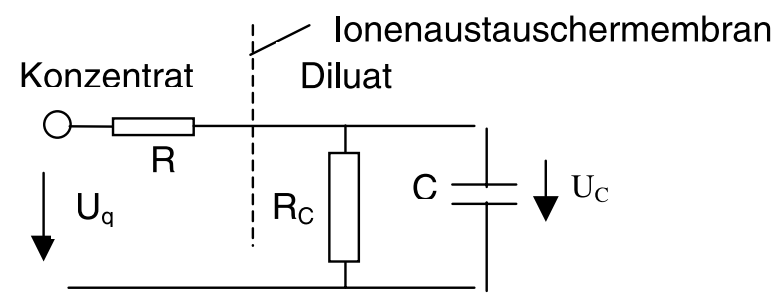

Abb. 2: Elektrisches Vergleichsschaltbild

$\mathrm{U}_{\mathrm{q}}$ steht für die angelegte Quellspannung. Die Konzentratkammer wird durch einen elektrischen Widerstand $\mathrm{R}$, die Diluatkammer durch einen Kondensator mit der Kapazität $\mathrm{C}$ und dem Rückdiffusionswiderstand $\mathrm{R}_{\mathrm{C}}$ simuliert. Diesem Vergleichsschaltbild liegt die Annahme zugrunde, dass der Widerstand in der Diluatkammer klein gegenuiber dem der Konzentratkammer ist, also vernachlässigt werden kann. $R$ ist durch Konvektion, $R_{C}$ dagegen durch elektrische Anziehung bestimmt.

Die Parallelschaltung mehrerer Kammerpaare $n_{M}$ zur Erhöhung des Durchsatzes stellt sich elektrisch als Reihenschaltung dar, wie in Abb. 3 dargestellt.

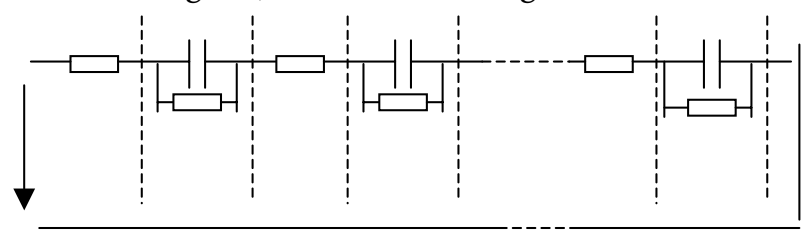

Abb. 3: Erweitertes elektrisches Vergleichsschaltbild

Für die Gesamtwiderstände aller Konzentrat- und Diluatkammern bzw. für die Gesamtkapazität gelten dann die Beziehungen:

$$
\begin{aligned}
& \mathrm{R}=\mathrm{R}_{0} \cdot \mathrm{n}_{\mathrm{M}} \\
& \mathrm{R}_{\mathrm{C}}=\mathrm{R}_{\mathrm{C} 0} \cdot \mathrm{n}_{\mathrm{M}} \\
& \mathrm{C}=\frac{\mathrm{C}_{\mathrm{O}}}{\mathrm{n}_{\mathrm{M}}}
\end{aligned}
$$


Herleitung der Differentialgleichung für die Spannung $\mathrm{U}_{\mathrm{c}}$, die am Kondensator anliegt:

$$
\begin{aligned}
& I=I_{R C}+I_{C} \text { mit } I_{R C}=\frac{U_{C}}{R_{C}}, \quad I_{C}=C \frac{d U_{C}}{d t} \\
& U_{q}=U_{R}+U_{C} \text { mit } U_{R}=I \cdot R=\left(\frac{U_{C}}{R_{C}}+C \frac{d U_{C}}{d t}\right) \cdot R \\
& U_{q}=\left(1+\frac{R}{R_{C}}\right) \cdot U_{C}+R \cdot C \frac{d U_{C}}{d t}
\end{aligned}
$$

Die Verbindung zum Stoffuibergang ergibt sich uiber die Faraday-Konstante, zunächst fuir $\mathrm{V}_{\text {Konzentrat }}=\mathrm{V}_{\text {Diluat }}=\mathrm{V}$, dem Vorlagevolumen

$$
\mathrm{I}_{\mathrm{C}}=\mathrm{C} \frac{\mathrm{dU_{C }}}{\mathrm{dt}}=\frac{\mathrm{n} \cdot \mathrm{F}}{\mathrm{n}_{\mathrm{M}}} \frac{\mathrm{dN}}{\mathrm{dt}}=\frac{\mathrm{n} \cdot \mathrm{F} \cdot \mathrm{V}}{\mathrm{n}_{\mathrm{M}}} \cdot \frac{\mathrm{dc}_{\text {Konzentrat }}}{\mathrm{dt}}
$$

mit $C \frac{d U_{C}}{d t}$, dem am Kondensator anliegenden $\frac{\mathrm{dN}}{\mathrm{dt}}$, dem übergehenden Stoffstrom

$\mathrm{C}_{\text {Konzentrat }}$, der Konzentration auf der Konzentratseite, im folgenden $c_{K}$

$\mathrm{F}=96490 \frac{\mathrm{As}}{\mathrm{eq}}$, der Faraday-Konstanten

n, der Ladungszahl

$\frac{\mathrm{dU}_{\mathrm{C}}}{\mathrm{dt}}=\frac{\mathrm{n} \cdot \mathrm{F} \cdot \mathrm{V}}{\mathrm{C} \cdot \mathrm{n}_{\mathrm{M}}} \cdot \frac{\mathrm{dc}_{\mathrm{K}}}{\mathrm{dt}}$

Durch Integration erhält man:

$U_{C}=\frac{n \cdot F \cdot V \cdot c_{K}}{C \cdot n_{M}}+K$ mit $K=0$, da für

$\mathrm{C}_{\mathrm{K}}=0 \quad \mathrm{U}_{\mathrm{C}}=0$ ist

Gl.(5) und (6) in Gl.(4) ergibt

$$
\frac{U_{q} \cdot C \cdot n_{M}}{n \cdot F \cdot V}=\left(1+\frac{R}{R_{C}}\right) \cdot c_{K}+R \cdot C \cdot \frac{d_{k}}{d t}
$$

und umgestellt

$$
\frac{\mathrm{dc}_{\mathrm{K}}}{\mathrm{dt}}=\frac{\mathrm{U}_{\mathrm{q}} \cdot \mathrm{n}_{\mathrm{M}}}{\mathrm{n} \cdot \mathrm{F} \cdot \mathrm{V} \cdot \mathrm{R}}-\left(\frac{1}{\mathrm{R} \cdot \mathrm{C}}+\frac{1}{\mathrm{C} \cdot \mathrm{R}_{\mathrm{C}}}\right) \cdot \mathrm{c}_{\mathrm{K}}
$$

Nimmt man weiterhin an, dass sich der Widerstand $\mathrm{R}$ umkehrt proportional zu der Leitfähigkeit $\kappa$ der Salzlösung verhält, und nähert man die Konzentrationsabhängigkeit der Leitfähigkeit durch die lineare Beziehung $\kappa=\mathrm{a} \cdot \mathrm{c}_{\mathrm{K}}$

mit $a=\frac{\kappa}{c_{k}}=\Lambda \cdot n=$ const $\left[\frac{\frac{\mathrm{s}}{\mathrm{cm}}}{\frac{\mathrm{mol}}{\mathrm{l}}}\right]$

und $\Lambda$ als Äquivalentleitfähigkeit an, lässt sich ein Wider- stand $\mathrm{R}_{\kappa}$ definieren, der leitfähigkeitsbestimmt ist:

$$
R_{\kappa}=\frac{L \cdot n_{M}}{\kappa \cdot A_{M}}=\frac{L \cdot n_{M}}{a \cdot c_{K} \cdot A_{M}}
$$

mit $A_{M}$, der aktiven Querschnittsfläche einer Membran und

L, der Breite einer Konzentratkammer.

Bezieht man $\mathrm{R}$ auf den Widerstand $\mathrm{R}_{\mathrm{K}}$, so erhält man einen dimensionslosen und konzentrationsunabhängigen Widerstand $\mathrm{r}$ :

$r=\frac{R}{R_{k}}=\frac{\text { Elektrischer Widers tan d bei Konvektion }}{\text { Elektrischer Widers tand aufgrund von Leitfähigkeit }}$

Einsetzen von (10) in (11) ergibt:

$R=r{ }^{*} R_{\kappa}=\frac{r \cdot n_{M} \cdot L}{a \cdot c_{K} \cdot A_{M}}$

und (12) in (8):

$\frac{d c_{K}}{d t}=\frac{U_{q} \cdot a \cdot c_{K} \cdot A_{M} \cdot n_{M}}{n \cdot F \cdot V \cdot r \cdot L \cdot n_{M}}-\left(\frac{a \cdot c_{K} \cdot A_{M}}{r \cdot C \cdot L \cdot n_{M}}+\frac{1}{C \cdot R_{C}}\right) \cdot c_{K}$
$\frac{d c_{K}}{d t}=-\frac{a \cdot A_{M}}{r \cdot C \cdot L \cdot n_{M}} \cdot c_{K}^{2}+\left(\frac{U_{q} \cdot a \cdot A_{M}}{n \cdot F \cdot V \cdot r \cdot L}-\frac{1}{C \cdot R_{C}}\right) \cdot c_{K}$

Die Lösung der Differentialgleichung vom Typ

$\frac{d c_{K}}{d t}=A \cdot c_{K}^{2}+B \cdot c_{K}$

lautet

$t-t_{0}=-\frac{2}{B} \cdot \operatorname{arctanh}\left(2 \cdot \frac{A}{B} c_{k}+1\right)$

mit $\mathbf{t}_{0}$, der Integrationskonstante

nach $c_{K}$ umgeformt

$\mathrm{C}_{\mathrm{K}}=-\frac{\mathrm{B}}{2 \cdot|\mathrm{A}|} \cdot\left[\tanh \left(-\frac{\left(\mathrm{t}-\mathrm{t}_{0}\right) \cdot \mathrm{B}}{2}\right)-1\right]$

In Abb. 4 ist der Funktionsverlauf zur Veranschaulichung dargestellt.

$c_{k}=-\frac{1}{2} \cdot 0.9 \cdot\left[\tanh \left(-\frac{(t-0) \cdot B}{2}\right)-1\right]$

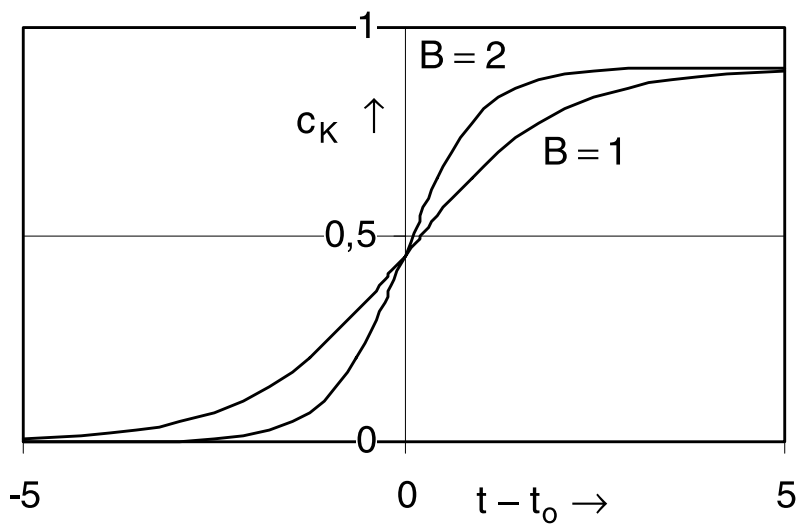

Abb. 4: Tangens hyperbolicus mit $B / I A I=0,9, t_{0}=0$ und dem Parameter $B=1$ und 2 . 
Wie zu erkennen ist, gibt B/IAI den gesamt durchfahrenden Konzentrationsbereich, beginnend bei 0 , an.

$\frac{B}{|A|}=c_{k \infty}$ mit $c_{k_{\infty}}$, der Konzentration,

die sich fuir $t \rightarrow \infty$ einstellt

Aus Gl.(13) und (14) ergibt sich

$\frac{B}{|A|}=\frac{U_{q} \cdot C \cdot n_{M}}{n \cdot F \cdot V}-\frac{r \cdot L \cdot n_{M}}{R_{C} \cdot a \cdot A_{M}}$

mit

$$
A=-\frac{a \cdot A_{M}}{r \cdot C \cdot L \cdot n_{M}}
$$

und

$$
\mathrm{B}=\frac{\mathrm{U}_{\mathrm{q}} \cdot \mathrm{a} \cdot \mathrm{A}_{\mathrm{M}}}{\mathrm{n} \cdot \mathrm{F} \cdot \mathrm{V} \cdot \mathrm{r} \cdot \mathrm{L}}-\frac{1}{\mathrm{R}_{\mathrm{C}} \cdot \mathrm{C}}
$$

Für den Fall, dass $V_{K} \neq V_{D}$ ist, ergeben sich für die Gl. (18), (19) und (20) folgende Veränderungen:

$$
\begin{aligned}
& \frac{B}{|A|}=\frac{U_{q} \cdot C \cdot n_{M}}{n \cdot F \cdot V_{K}}-\frac{r \cdot L \cdot n_{M} \cdot V_{D}}{R_{C} \cdot a \cdot A_{M} \cdot V_{K}} \\
& A=-\frac{a \cdot A_{M} \cdot V_{K}}{r \cdot C \cdot L \cdot n_{M} \cdot V_{D}} \\
& B=\frac{U_{q} \cdot a \cdot A_{M}}{n \cdot F \cdot V_{D} \cdot r \cdot L}-\frac{1}{R_{C} \cdot C}
\end{aligned}
$$

Der erste Term in Gl. (18a) gibt die erreichbare Konzentration für den idealen Fall $R_{C}=\infty$ an. In diesem Falle würde sich die gesamte Stoffmenge für $\mathrm{t} \rightarrow \infty$ auf der Konzentratseite befinden, d. h. $\mathrm{c}_{\text {Diluat }}=0$

$$
c_{K \max }=\frac{U_{q} \cdot C \cdot n_{M}}{n \cdot F \cdot V_{K}}=\frac{N_{G e s}}{V_{K}}=\frac{c_{K}\left(t_{1}\right) \cdot V_{k}+c_{D}\left(t_{1}\right) \cdot V_{D}}{V_{K}}
$$

mit $t_{1}$, einem beliebigen Zeitpunkt.

Aus Gl. (21) lässt sich die Kapazität C berechnen.

$$
\mathrm{C}=\frac{\mathrm{N}_{\mathrm{Ges}} \cdot \mathrm{n} \cdot \mathrm{F}}{\mathrm{U}_{\mathrm{q}} \cdot \mathrm{n}_{\mathrm{M}}}\left[\frac{\mathrm{As}}{\mathrm{V}}\right]
$$

Der zweite Term in Gl. (18a) ist demnach

$$
\mathrm{C}_{\mathrm{KVerlust}}=\mathrm{C}_{\mathrm{Kmax}}-\mathrm{C}_{\mathrm{K}_{\infty}}=\frac{\mathrm{V}_{\mathrm{D}}}{\mathrm{V}_{\mathrm{K}}} \mathrm{C}_{\mathrm{D}_{\infty}}=\frac{\mathrm{r} \cdot \mathrm{L} \cdot \mathrm{n}_{\mathrm{M}} \cdot \mathrm{V}_{\mathrm{D}}}{\mathrm{R}_{\mathrm{C}} \cdot \mathrm{a} \cdot \mathrm{A}_{\mathrm{M}} \cdot \mathrm{V}_{\mathrm{K}}}
$$

mit $c_{D \infty}$, der Diluatendkonzentration für $t \rightarrow \infty$. Damit ist

$$
\frac{B}{|A|}=c_{K \infty}-\frac{V_{D}}{V_{K}} \cdot c_{D \infty}=\frac{N_{G e s}}{V_{K}}-\frac{r \cdot L \cdot n_{M} \cdot V_{D}}{R_{C} \cdot a \cdot A_{M} \cdot V_{K}}
$$

Geht man davon aus, dass der Diffusionskoeffizient $D_{M}$ durch die Membran in Richtung Diluat sehr viel kleiner als die Stoffuibergangskoeffizienten $\beta$ an der Membran sind und dass $c_{\mathrm{Kmax}}>>\mathrm{c}_{\mathrm{KVerlust}}$ ist, lässt sich folgender Zusammenhang für $\mathrm{R}_{\mathrm{C}}$ herstellen:

$$
\begin{aligned}
& R_{C}=\frac{U_{q}}{I}=\frac{U_{q}}{\dot{N} \cdot n \cdot F}=\frac{U_{q}}{d_{M} \cdot A_{M} \cdot C_{K \max } \cdot n \cdot F} \\
& \text { mit } \dot{N}=\frac{D_{M} \cdot A_{M}}{S_{M}} \cdot\left(c_{K_{\infty}}-C_{D \infty}\right)=d_{M} \cdot A_{M} \cdot C_{K \max }
\end{aligned}
$$

mit $\dot{N}$, dem Stoffstrom, der für t in eine Diluatkammer zurüickströmt,

$\mathrm{s}_{\mathrm{M}}$, der Dicke der Membran,

$\mathrm{d}_{\mathrm{M}}$, dem spezifischen Diffusionskoeffizienten,

eingesetzt in Gl. (23) ergibt die Berechnungsgleichung fuir $\mathrm{C}_{\mathrm{D} \infty}$ :

$$
c_{D \infty}=\frac{r \cdot d_{M} \cdot c_{K \max } \cdot n \cdot F \cdot L \cdot n_{M}}{U_{q} \cdot a}
$$

Die Integrationskonstante $t_{0}$ lässt sich aus der Beziehung $\mathrm{t}-\mathrm{t}_{0}=0$ bei $0.5 \cdot \mathrm{c}_{\mathrm{K}_{\infty}}$ bestimmen.

$$
\text { Für } \quad \begin{aligned}
& \mathrm{c}_{\mathrm{KAnfang}}<\frac{\mathrm{c}_{\mathrm{K}_{\infty}}}{2} \text { ist } \mathrm{t}_{0}>0 ; \\
& \mathrm{C}_{\text {KAnfang }}=\frac{\mathrm{c}_{\mathrm{K}_{\infty}}}{2} \text { ist } \mathrm{t}_{0}=0 ; \\
& \mathrm{c}_{\text {KAnfang }}>\frac{\mathrm{c}_{\mathrm{K}_{\infty}}}{2} \text { ist } \mathrm{t}_{0}<0
\end{aligned}
$$

Die Gleichungen (16), (20a), (24) und (27) enthalten die wichtigsten prozess- und apparatespezifischen Größen.

Experimentell bestimmt werden muss lediglich noch die Abhängigkeit des dimensionslosen Widerstandes $r$ von der Strömungsgeschwindigkeit sowie der spez. Diffusionskoeffizient $\mathrm{d}_{\mathrm{M}}$.

Üblicherweise werden aus Gründen der Messgenauigkeit Messwerte in der Form $c_{\text {Diluat }}=\mathrm{f}(\mathrm{t})$ aufgenommen. Die Umrechnung in $\mathrm{c}_{\mathrm{K}}$ erfolgt mit Gl. (21) über

$\mathrm{C}_{\mathrm{K}}=\mathrm{C}_{\mathrm{Kmax}}-\frac{\mathrm{V}_{\mathrm{D}}}{\mathrm{V}_{\mathrm{K}}} \cdot \mathrm{C}_{\mathrm{D}}$

Der dimensionslose Widerstand $r$ lässt sich über B bestimmen (Gl. (15), (17), (20a))

$$
\mathrm{B}=-\frac{2}{\left(\mathrm{t}-\mathrm{t}_{0}\right)} \cdot \operatorname{arctanh}\left(1-\frac{2 \cdot \mathrm{c}_{\mathrm{K}}}{\mathrm{C}_{\mathrm{k} \infty}}\right)=\frac{\mathrm{U}_{\mathrm{q}} \cdot \Lambda \cdot \mathrm{A}_{\mathrm{M}}}{\mathrm{F} \cdot \mathrm{V}_{\mathrm{D}} \cdot \mathrm{r} \cdot \mathrm{L}}
$$

(der zweite Term in Gl. (20a) kann vernachlässigt werden, wenn $c_{K \max }>>c_{K V e r l u s t}$ ist), der spez. Diffusionskoeffizient $d_{M}$ über $c_{D}$ nach Gl. (27).

\section{Messergebnisse}

Die Größen B und r lassen sich mit Hilfe von Messdaten errechnen. Nach Gleichung (29) benötigt man dazu den zeitlichen Konzentrationsverlauf für das Konzentrat, $c_{K}=f(t)$, der sich experimentell ermitteln lässt. 
Die nachfolgend dargestellten Messergebnisse wurden mit einer Apparatur, bestehend aus 20 Kammerpaaren, ausgestattet mit Membranen der Firma Stantech vom Typ AMX und CMX 16x16 cm, durchgefuihrt. Die Kammernbreite betrug $0,2 \mathrm{~cm}$, die aktive Membranfläche $65 \mathrm{~cm}^{2}$. Als Salz kam Na${ }_{2} \mathrm{SO}_{4}$ zur Anwendung, die Ausgangskonzentration betrug für Diluat und Konzentrat $0,03 \mathrm{~mol} / \mathrm{l}$, was einer einprozentigen Lösung hydratisierten Salzes entspricht.

Die Volumenströme wurden zur Vermeidung von Leckageverlusten auf beiden Seiten gleich gehalten. Die gleiche Konzentration wurde zur Elektrodenspuilung verwendet, der Volumenstrom betrug für Diluat, Konzentrat und Elektrodenspülung jeweils 200 l/h, die Quellspannung $40 \mathrm{~V}$ und die Vorlagevolumen für die drei Kreisläufe jeweils $5 \mathrm{l}$. Als mittlere Aquivalentleitfähigkeit für $\mathrm{Na}_{2} \mathrm{SO}_{4}$ wurde $0,1175 \frac{\mathrm{S} \cdot \mathrm{l}}{\mathrm{mol} \cdot \mathrm{cm}}$ verwendet.

Die Ergebnisse der mit den Messergebnissen durchgefuihrten Berechnungen sind in den Abbildungen 5 und 6 dargestellt.

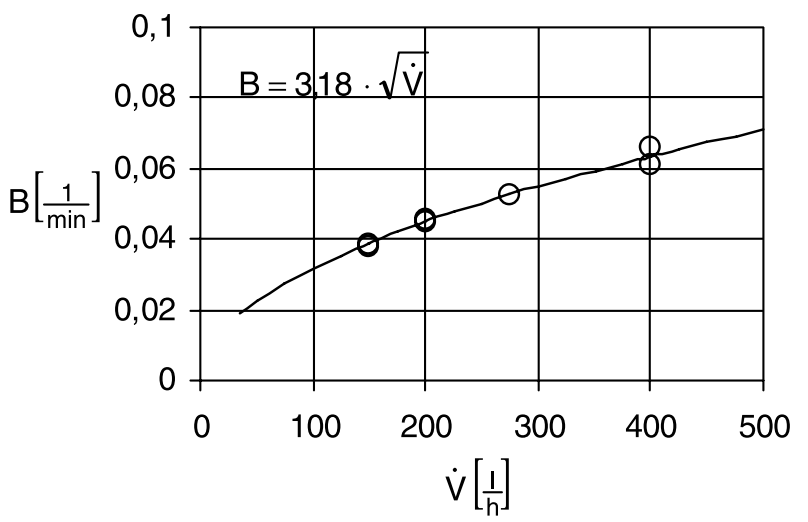

Abb. 5: B als Funktion des Diluat- und Konzentratvolumenstromes

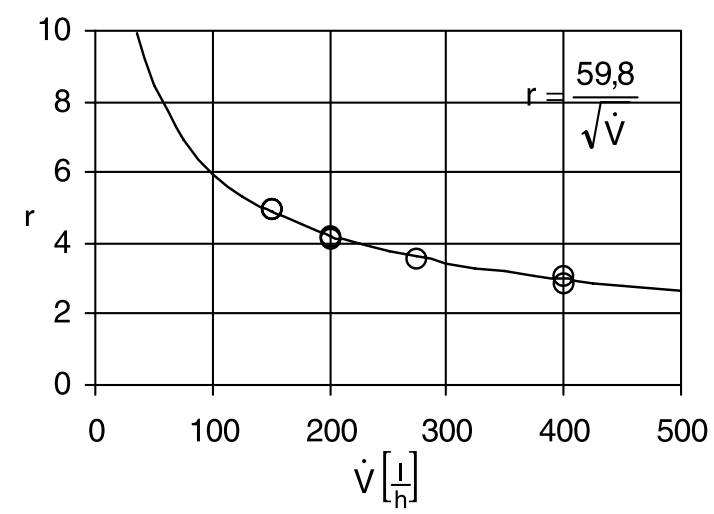

Abb. 6: Der dimensionslose Widerstand $r$ als Funktion des Volumenstromes

Theoretisch, d. h. unabhängig von den durchgefuihrten Messungen muss der dimensionslose Widerstand $r$ für kleine Volumenströme sehr groß sein, weil die Konvektion dann schwach und der Zähler in Gleichung (11) somit groß ist. Mit steigendem Volumenstrom fällt der Zähler in Gleichung (11) im Extremfall bis zu r = 1, d. h. der Widerstand entspricht dann dem mit Leitfähigkeit und Geometrie berechneten Wert. Der Leitfähigkeitswiderstand $R_{\kappa}$ wurde mit Hilfe von Wechselspannung bestimmt, um Polarisation zu vermeiden und den Übergangswiderstand zu minimieren, das Konzentrationsprofil zwischen beiden Elektroden ist dabei kolben- förmig. Diesen Zustand erreicht man in der mit Gleichspannung betriebenen Elektrodialyse nur bei technisch nicht realisierbarer Turbulenz, da die hohen Volumenströme zu nicht handhabbaren Drücken führen würden.

Die aus Messergebnissen errechneten und in Abb. 5 und 6 dargestellten Größen zeigen den erwarteten Verlauf. $B$ steigt und $r$ fällt erwartungsgemäß mit steigenden Volumenströmen. Beide Größen verhalten sich gegenläufig gemäß Gleichung (29).

Mit diesem Modell können Messergebnisse modelliert werden. So z. B. zeigen Abb. 7 und 8 ein typisches Ergebnis für eine solche Modellierung. In Abb. 7 ist B, in Abb. 8 die gemessene und die mit diesem Modell errechnete Diluatkonzentration über der Zeit dargestellt. Wie man sieht, sank die Diluatkonzentration im Laufe des knapp vierstündigen Versuches von $0,015 \mathrm{~mol} / \mathrm{l}$ auf unter 0,00005 mol/l. Die gemessenen Werte werden durch die errechnete Ausgleichskurve sehr gut wiedergegeben. Als Funktion für die Berechnung wurde dabei verwendet:

$$
\mathrm{C}_{\mathrm{D}}=\frac{\mathrm{V}_{\mathrm{K}}}{\mathrm{V}_{\mathrm{D}}}\left[\mathrm{C}_{\mathrm{Kmax}}+\frac{\mathrm{C}_{\mathrm{K}_{\infty}}}{2}\left\{\tanh \left(-\frac{\mathrm{B} \cdot\left(\mathrm{t}-\mathrm{t}_{0}\right)}{2}\right)-1\right\}\right]
$$

mit $\mathrm{C}_{\mathrm{Kmax}}=\frac{\mathrm{N}_{\text {ges }}}{\mathrm{V}_{\mathrm{K}}} ; \mathrm{N}_{\text {ges }}$ gesamte Stoffmenge im Diluat und $\mathrm{C}_{\mathrm{K}_{\infty}}=$ maximal erreichte Konzentrat-Konzentration.

Gleichung (30) erhält man aus Gleichung (28) mit $C_{K}$ nach Gl. (16) und B nach Gl. (29).

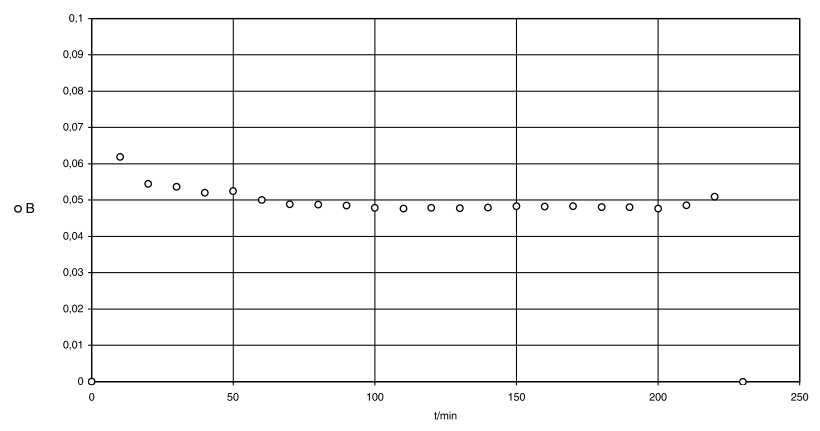

Abb. 7: Abhängigkeit des aus Messwerten ermittelten Faktors B von der Zeit

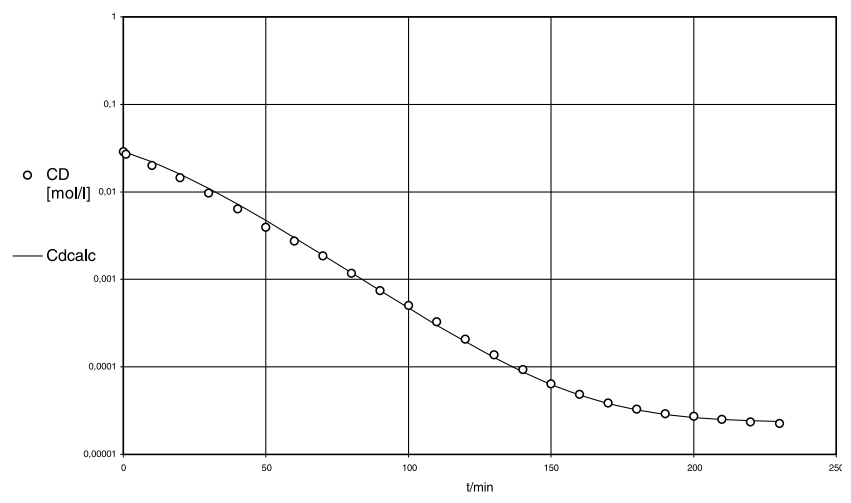

Abb. 8: Abhängigkeit der gemessenen und der nach Gl. (30) errechneten Konzentration $\mathrm{C}_{\mathrm{D}}$ von der Zeit 


\section{Zusammenfassung}

In dieser Arbeit wird ein theoretisches Modell für die Elektrodialyse mit Ionenaustauschermembranen in Anlehnung an den elektrischen Kondensator entwickelt und anhand von experimentellen Messwerten, die mit einer vorhandenen Apparatur gewonnen wurden, überprüft. Es zeigte sich, dass die aus Messwerten bestimmten Parameter B und $r$ des Modells bei Variation des Volumenstroms den erwarteten Trend zeigen und dass sich die Zeitabhängigkeit der Konzentrationen, im dargestellten Beispiel die Diluatkonzentration, mit diesem Modell im Rahmen der Messgenauigkeit nahezu exakt beschreiben lässt.

Das entwickelte Modell soll in Folgearbeiten auf seine Tauglichkeit auch für andere Anwendungen der Elektrolyse mit Ionenaustauschermembranen untersucht und gegebenenfalls weiterentwickelt werden.

Für die Überlassung der Versuchsapparatur sind wir Herrn Prof. Dr. rer. nat. Franz Wildenauer zu Dank verpflichtet.

\section{Autoren}

Prof. Dr.-Ing. Josef Schmadl

Dipl.-Ing. Jens-Uwe Gerking

Technische Fachhochschule Wildau

Technikum für Thermische Verfahrenstechnik

Telefon +493375 508-110

E-Mail: jschmadl@igw.tfh-wildau.de 